\title{
Upper Semicontinuity of Trajectory Attractors for 3D Incompressible Navier-Stokes Equation
}

\author{
Yuming Qin ${ }^{1}\left[\right.$. Xiuqing Wang ${ }^{2}$
}

Published online: 13 November 2019

(C) The Author(s) 2019

\begin{abstract}
In this paper, we first establish the existence of a trajectory attractor for the NavierStokes-Voight (NSV) equation and then prove upper semicontinuity of trajectory attractors of 3D incompressible Navier-Stokes equation when 3D NSV equation is considered as a perturbative equation of 3D incompressible Navier-Stokes equation.
\end{abstract}

Keywords Navier-Stokes-Voight equation · Navier-Stokes equation · Trajectory attractors

Mathematics Subject Classification 35M13 $\cdot 35 \mathrm{Q} 35$

\section{Introduction}

It is very significant and difficult to study the uniqueness and asymptotic behaviour of the evolution equations and their long-time behavior of solutions can be described by attractors. But, we all know that the uniquences of weak solutions for the 3D Navier-Stokes equation is still open until now. To this end, Chepyzhov and Vishik in [6] proposed the trajectory attractor theory, which can describe the long-time behavior of solutions whose uniqueness is not known. We study the relationship between the following 3D Navier-Stokes equation:

Yuming Qin

yuming_qin@hotmail.com

Xiuqing Wang

dqwangdq@foxmail.com

1 College of Sciences, Institute for Nonlinear Sciences, Donghua University, Shanghai 201620,

People's Republic of China

2 College of Information Science and Technology, Donghua University, Shanghai 201620,

People's Republic of China 


$$
\left\{\begin{array}{l}
u_{t}-v \Delta u+(u \cdot \nabla) u+\nabla p=g(x), \quad x \in \Omega, \quad t \geq 0, \\
\nabla \cdot u=0
\end{array}\right.
$$

and the 3D Navier-Stokes-Voight equation:

$$
\left\{\begin{array}{l}
u_{t}-\alpha^{2} \triangle u_{t}-v \Delta u+(u \cdot \nabla) u+\nabla p=g(x), \quad x \in \Omega, \quad t \geq 0, \\
\nabla \cdot u=0
\end{array}\right.
$$

subjecting to the noslip boundary condition

$$
\left.u\right|_{\partial \Omega}=0,
$$

and the initial conditions

$$
u(x, 0)=u_{0}, \quad x \in \Omega
$$

where $\Omega \subseteq \mathbb{R}^{3}$ is a smooth bounded domain, $u(x, t)=\left(u^{1}(x, t), u^{2}(x, t), u^{3}(x, t)\right)$ is the velocity vector, $p(x, t)$ is the pressure function, and $g=\left(g^{1}(x), g^{2}(x), g^{3}(x)\right)$ is an external term, $v$ is a positive viscous constant.

There has been many literature on the trajectory attractors [3,7,14-17,19]. First, Chepyzhow et al. [4,5] study the following 3D Navier-Stokes- $\alpha$ model,

$$
\left\{\begin{array}{l}
v_{t}-v \Delta v+(u \cdot \nabla) v+\nabla P=g(x), \quad x \in \Omega, \quad t \geq 0, \\
v=u-\alpha^{2} \Delta u, \quad \nabla \cdot u=0
\end{array}\right.
$$

and showed that the trajectory attractor of the Navier-Stokes- $\alpha$ model converges to the trajectory attractor of the 3D Navier-Stokes system as $\alpha \rightarrow 0^{+}$. The difference between [4] and [5] is that they chose different trajectory spaces.

There are also a lot of literature on Navier-Stokes- $\alpha$ model $[1,2,9,10,18]$. Zelati and Gal [21] proved the existence of global and exponential attractors, then they prove the convergence of the (strong) global attractor of the 3D Navier-Stokes-Voight model to the (weak) global attractor of the 3D Navier-Stokes equation, i.e., they prove the convergence of the (strong) global attractor of the 3D Navier-Stokes-Voight model to the trajectory attractor of the 3D Navier-Stokes equation. But, they did not prove upper semicontinuity of trajectory attractors. In addition, trajectory attractor was also obtained for the fluid dynamics systems such as the MHD system [8], liquid crystal flow [12], binary fluid mixtures [13], and Cahn-Hilliard-Navier-Stokes equations [11]. These 3D systems all have trajectory attractors but not necessarily have global attractors. Zhao and Zhou [22] first proposed the concept of pullback trajectory attractors, and proved the existence of pullback trajectory attractors to 3D incompressible non-Newtonian fluid.

\section{Preliminaries}

In this section, we will first introduce some Sobolev spaces which will be used, and their dual spaces. It is also necessary to introduce some notations related to trajectory 
attractors. At last, we give a result on the trajectory attractors of 3D incompressible Navier-Stokes equation.

\subsection{Trajectory Space}

We first introduce some functional spaces and operators. Set

$$
\begin{aligned}
& \mathcal{V}=\left\{\varphi(x)=\left(\varphi^{1}(x), \varphi^{2}(x), \varphi^{3}(x)\right) \in\left(C_{0}^{\infty}(\bar{\Omega})\right)^{3}, \quad \nabla \cdot \varphi=0,\left.\quad \varphi\right|_{\partial \Omega}=0\right\} \\
& H=\text { closure of } \mathcal{V} \text { in }\left(L^{2}(\Omega)\right)^{3} \text { with norm }|\cdot|, \quad H^{\prime} \text { is the dual space of } H ; \\
& V=\text { closure of } \mathcal{V} \text { in }\left(H^{1}(\Omega)\right)^{3} \text { with norm }\|\cdot\|, \quad V^{\prime} \text { is the dual space of } V,
\end{aligned}
$$

where $(\cdot, \cdot),\langle\cdot, \cdot\rangle$ denote the inner products in $H$ and in $V$ respectively. For any $v \in V^{\prime}$, the expression $\langle u, v\rangle$ means the value of the functional $v$ on a vector $u \in V$. In the sequel, we identify $H$ with its dual and we have the following inclusions,

$$
D(A) \subset V \subset H=H^{\prime} \subset V^{\prime} \subset D(A)^{\prime} .
$$

We set $H^{\eta}=(-\triangle)^{\frac{\eta}{2}} H$ and use $H^{-\eta}$ denote the dual space of $H^{\eta}$. Clearly, the embedding $H \hookrightarrow H^{-\eta}$ is compact.

The operator $P:\left[L^{2}(\Omega)\right]^{3} \rightarrow H$ denotes the orthogonal projector, and $A$ is the Stokes operator with the domain $D(A)=\left(H^{2}(\Omega)\right)^{3} \cap V$, the operator $A$ is self-adjoint and positive,

$$
A u=-P \triangle u \text {. }
$$

The bilinear operator $B(u, u)$ on $V \times V \rightarrow V^{\prime}$ is defined as

$$
\langle B(u, v), w\rangle=b(u, v, w)
$$

and the trilinear form $b: V \times V \times V$,

$$
b(u, v, w)=\int_{\Omega}(u \cdot \nabla) v \cdot w d x=\sum_{i, j=1}^{3} \int_{\Omega} u_{i} \frac{\partial v_{j}}{\partial x_{i}} w_{j} d x
$$

satisfies

$$
b(u, v, w)=-b(u, w, v), \quad b(u, v, v)=0, \quad u, v, w \in V,
$$

and

$$
|\langle B(u, v), w\rangle| \leq c|u|_{3}|v||A w|, \quad\|B(u, v)\|_{D(A)^{\prime}} \leq c|u|_{3}|v| .
$$


We recall the Poincaré inequality,

$$
|u| \leq \frac{1}{\sqrt{\lambda_{1}}}\|u\|, \quad \forall u \in V,
$$

where $\lambda_{k}(k=1,2, \ldots)$ is the eigenvalue of Stokes operator $A$, and $\lambda_{k}$ satisfies

$$
0<\lambda_{1}<\lambda_{2}<\cdots<\lambda_{k}, \quad \lambda_{k} \rightarrow+\infty, k \rightarrow \infty
$$

We consider the spaces $\mathcal{F}_{+}^{b}$ defined by

$$
\mathcal{F}_{+}^{b}=\left\{z(\cdot) \mid z(\cdot) \in L_{b}^{2}\left(\mathbb{R}_{+} ; V\right) \cap L^{\infty}\left(\mathbb{R}_{+} ; H\right), \partial_{t} z(\cdot) \in L_{b}^{2}\left(\mathbb{R}_{+} ; D(A)^{\prime}\right)\right\},
$$

with norm

$$
\|z\|_{\mathcal{F}_{+}^{b}}=\|z\|_{L_{b}^{2}\left(\mathbb{R}_{+} ; V\right)}+\|z\|_{L^{\infty}\left(\mathbb{R}_{+} ; H\right)}+\left\|\partial_{t} z\right\|_{L_{b}^{2}\left(\mathbb{R}_{+} ; D(A)^{\prime}\right)}
$$

where

$$
\begin{aligned}
& \|z\|_{L_{b}^{2}\left(\mathbb{R}_{+} ; V\right)}^{2}=\sup _{t \geq 0} \int_{t}^{t+1}\|z(s)\|^{2} d s, \quad\|z\|_{L^{\infty}\left(\mathbb{R}_{+} ; H\right)}=\text { ess } \sup _{t \geq 0}|z(t)|, \\
& \left\|\partial_{t} z\right\|_{L_{b}^{2}\left(\mathbb{R}_{+} ; D(A)^{\prime}\right)}^{2}=\sup _{t \geq 0} \int_{t}^{t+1}\left\|\partial_{t} z(s)\right\|_{D(A)^{\prime}}^{2} d s .
\end{aligned}
$$

We know that $\mathcal{F}_{+}^{b}$ with its norm $\|\cdot\|_{\mathcal{F}_{+}^{b}}$ is a Banach space. Similarly, we define the space

$$
\mathcal{F}_{+}^{\text {loc }}=\left\{z(\cdot) \mid z(\cdot) \in L_{\text {loc }}^{2}\left(\mathbb{R}_{+} ; V\right) \cap L_{\text {loc }}^{\infty}\left(\mathbb{R}_{+} ; H\right), \partial_{t} z(\cdot) \in L_{\text {loc }}^{2}\left(\mathbb{R}_{+} ; D(A)^{\prime}\right)\right\},
$$

and we define a topology $\Theta_{+}^{l o c}$ on $\mathcal{F}_{+}^{\text {loc }}$, then we consider a topology sequence $\left\{z_{n}(\cdot)\right\} \subset$ $\mathcal{F}_{+}^{l o c}, z_{n} \rightarrow z$ in the topology $\Theta_{+}^{l o c}$, i.e.,

$z_{n} \rightarrow z$, weakly in $L^{2}(0, M ; V)$, and weakly-star in $L^{\infty}(0, M ; H), n \rightarrow \infty$, $\partial_{t} z_{n} \rightarrow \partial_{t} z$, weakly in $L^{2}\left(0, M ; D(A)^{\prime}\right), n \rightarrow \infty$.

Let $\{T(h) \mid \geq 0\}$ denote the time translation operator acting on the trajectory space,

$$
T(h) z(t)=z(t+h) .
$$

\subsection{Some Useful Lemmas}

Lemma 2.1 [20] Let $y(t) \in C^{1}\left[t_{0}, t_{1}\right], y \geq 0$ and the following inequality

$$
y^{\prime}(t)+k y(t) \leq h(t)
$$


holds with $k>0, h(t) \in C\left[t_{0}, t_{1}\right]$, then

$$
y(t) \leq y(0) e^{-k t}+\int_{0}^{t} e^{-k(t-s)} h(s) d s .
$$

In particular, if $h(t)=C$, then

$$
y(t) \leq y(0) e^{-k t}+C k^{-1}
$$

Lemma 2.2 [6] Suppose that $E_{1} \hookrightarrow E \subset E_{0}$, where $E$ is a Banach space, and the embedding $E_{1} \hookrightarrow E$ is compact. Set the space

$$
\begin{aligned}
& W_{p_{1}, p_{0}}\left(0, T ; E_{1}, E_{0}\right) \\
& \quad=\left\{\psi(s), s \in[0, T] \mid \psi(s) \in L^{p_{1}}\left(0, T ; E_{1}\right), \psi^{\prime}(s) \in L^{p_{0}}\left(0, T ; E_{0}\right)\right\},
\end{aligned}
$$

with the norm

$$
\|\psi\|_{W_{p_{1}, p_{0}}\left(0, T ; E_{1}, E_{0}\right)}=\left(\int_{0}^{T}\|\psi(s)\|_{E_{1}}^{p_{1}} d s\right)^{\frac{1}{p_{1}}}+\left(\int_{0}^{T}\left\|\psi^{\prime}(s)\right\|_{E_{0}}^{p_{0}} d s\right)^{\frac{1}{p_{0}}} .
$$

We assume that $p_{1} \geq 1$ and $p_{0} \geq 1$. Then the following embedding is compact:

$$
W_{p_{1}, p_{0}}\left(0, T ; E_{1}, E_{0}\right) \hookrightarrow L^{p_{1}}(0, T ; E) .
$$

Moreover, when $p_{1}=\infty$, then the following embedding is compact:

$$
W_{\infty, p_{0}}\left(0, T ; E_{1}, E_{0}\right) \hookrightarrow C(0, T ; E) .
$$

Lemma 2.3 [4] For any $f(t) \in D(A)^{\prime}$, assume the operator $A$ is self-adjoint and positive, then the following inequality holds:

$$
\|f\|_{D(A)^{\prime}}^{2} \leq\left\|\left(1+\alpha^{2} A\right) f\right\|_{D(A)^{\prime}}^{2} .
$$

Lemma 2.4 [4] For any $f(t) \in L^{2}\left(0, M ; D(A)^{\prime}\right)$, assume the operator $A$ is selfadjoint and positive, then there holds that

$$
\lim _{\alpha \rightarrow 0^{+}}\left\|\left(1+\alpha^{2} A\right) f(t)\right\|_{L^{2}\left(0, M ; D(A)^{\prime}\right)}=\|f(t)\|_{L^{2}\left(0, M ; D(A)^{\prime}\right)} .
$$

\subsection{Trajectory Attractors of 3D Incompressible Navier-Stokes Equation}

In this subsection, we will present the theory of trajectory attractors of 3D incompressible Navier-Stokes equation, which can be found in [6]. With the orthogonal projector P, 3D incompressible Navier-Stokes system can be rewritten as

$$
\left\{\begin{array}{l}
\partial_{t} u+v A u+B(u, u)=g(x), \quad x \in \Omega, \quad t>0 \\
\nabla \cdot u=0,\left.\quad u\right|_{\partial \Omega}=0, \quad u(x, 0)=u_{0} \in H
\end{array}\right.
$$


Lemma 2.5 If $g \in H, u_{0} \in H$, and $M>0$, then problem (2.4) admits a weak solution $u(t) \in L^{\infty}(0, M ; H) \cap L^{2}(0, M ; V)$ such that $u(0)=u_{0}$ and there holds that

$$
\frac{1}{2} \frac{d}{d t}|u(t)|^{2}+v\|u(t)\|^{2}=(g, u(t)), \quad \forall t \in[0, M] .
$$

Moreover, for any $\psi(t) \in C_{0}^{\infty}(0, M)$, there holds that

$$
-\frac{1}{2} \int_{0}^{M}|u(t)|^{2} \psi^{\prime}(t) d t+v \int_{0}^{M}\|u(t)\|^{2} \psi(t) d t \leq \int_{0}^{M}(g, u(t)) \psi^{\prime}(t) d t .
$$

Definition 2.6 The trajectory space $\mathcal{K}_{0}$ is the union of weak solutions $u(t)$ of problem (2.4) with an arbitrary $u_{0} \in H$, i.e., $u(t)$ satisfies (2.5)-(2.6). $\mathcal{K}_{0}$ is called the trajectory space of problem (2.4).

Lemma 2.7 If $g \in H$, then the equation (2.4) has a trajectory attractor $\mathcal{O}_{0}$.

Proof We refer to [6] for its proof.

\section{Trajectory Attractors of 3D Navier-Stokes-Voight Equation}

The 3D Navier-Stokes-Voight system can be rewritten as

$$
\left\{\begin{array}{l}
\left(1+\alpha^{2} A\right) \partial_{t} u+v A u+B(u, u)=g(x), \quad x \in \Omega \\
\nabla \cdot u=0,\left.\quad u\right|_{\partial \Omega}=0, \quad u(x, 0)=u_{0} \in V
\end{array}\right.
$$

Theorem 3.1 If $g \in H, u_{0} \in V$, and $M>0$, then problem (3.1) admits a weak solution $u(t) \in L^{\infty}(0, M ; H) \cap L^{2}(0, M ; V)$ such that $u(0)=u_{0}$ and there holds that

$$
\frac{1}{2} \frac{d}{d t}\left(|u(t)|^{2}+\alpha^{2}\|u(t)\|^{2}\right)+v\|u(t)\|^{2}=(g, u(t)), \quad \forall t \in[0, M]
$$

Moreover, for any $\psi(t) \in C_{0}^{\infty}(0, M)$, there holds that

$$
\begin{aligned}
& -\frac{1}{2} \int_{0}^{M}\left(|u(t)|^{2}+\alpha^{2}\|u(t)\|^{2}\right) \psi^{\prime}(t) d t \\
& \quad+v \int_{0}^{M}\|u(t)\|^{2} \psi(t) d t=\int_{0}^{M}(g, u(t)) \psi^{\prime}(t) d t .
\end{aligned}
$$

Definition 3.1 The trajectory space $\mathcal{K}_{\alpha}^{+}$is the union of weak solutions $u(t)$ of problem (3.1) with an arbitrary $u_{0} \in V$, i.e., $u(t)$ satisfies (3.2)-(3.3). $\mathcal{K}_{\alpha}^{+}$is called the trajectory space of problem (3.1).

We consider the time translation semigroup $\{T(h)\}$ acting on $\mathcal{K}_{\alpha}^{+}$. 
Proposition 3.2 The trajectory space $\mathcal{K}_{\alpha}^{+}$is invariant with the time translation semigroup $\{T(h) \mid h \geq 0\}$ :

$$
T(h) \mathcal{K}_{\alpha}^{+} \subseteq \mathcal{K}_{\alpha}^{+}, \forall h \geq 0
$$

Proof For any $u(t) \in \mathcal{K}_{\alpha}^{+}$,

$$
T(h) u(t)=u(h+t) .
$$

Obviously, $u(h+t)$ is also a weak solution of problem (3.1), hence $u(h+t) \in \mathcal{K}_{\alpha}^{+}$.

Lemma 3.2 If $g \in H$ and $u(t)$ is a weak solution of problem (3.1), then $u(t)$ satisfies (3.2), and the following inequalities hold:

$$
\begin{aligned}
& |u(t)|^{2}+\alpha^{2}\|u(t)\|^{2} \leq\left(|u(0)|^{2}+\alpha^{2}\|u(0)\|^{2}\right) e^{-\frac{v \lambda_{1}}{1+\lambda_{1} \alpha^{2}} t} \\
& +\frac{\left(1+\lambda_{1} \alpha^{2}\right)|g|^{2}}{v^{2} \lambda_{1}^{2}}, \\
& v \int_{t}^{t+1}\|u(s)\|^{2} d s \leq\left(|u(0)|^{2}+\alpha^{2}\|u(0)\|^{2}\right) e^{-\frac{v \lambda_{1}}{1+\lambda_{1} \alpha^{2}} t}+\frac{\left(1+\lambda_{1} \alpha^{2}\right)|g|^{2}}{v^{2} \lambda_{1}^{2}} \\
& \quad+\frac{|g|^{2}}{v \lambda_{1}} .
\end{aligned}
$$

Proof By the Cauchy inequality, it follows that

$$
(g, u(t)) \leq \frac{v}{2}\|u(t)\|^{2}+\frac{1}{2 v \lambda_{1}}|g|^{2} .
$$

Then inserting (3.6) into (3.2) yields

$$
\frac{d}{d t}\left(|u(t)|^{2}+\alpha^{2}\|u(t)\|^{2}\right)+v\|u(t)\|^{2} \leq \frac{1}{v \lambda_{1}}|g|^{2} .
$$

Using the Poincaré inequality (2.3), we get

$$
|u(t)|^{2}+\alpha^{2}\|u(t)\|^{2} \leq \frac{1}{\lambda_{1}}\|u(t)\|^{2}+\alpha^{2}\|u(t)\|^{2}=\frac{1+\lambda_{1} \alpha^{2}}{\lambda_{1}}\|u(t)\|^{2},
$$

i.e.,

$$
\frac{\lambda_{1}}{1+\lambda_{1} \alpha^{2}}\left(|u(t)|^{2}+\alpha^{2}\|u(t)\|^{2}\right) \leq\|u(t)\|^{2} .
$$

Inserting (3.8) into (3.7), we obtain

$$
\frac{d}{d t}\left(|u(t)|^{2}+\alpha^{2}\|u(t)\|^{2}\right)+\frac{v \lambda_{1}}{1+\lambda_{1} \alpha^{2}}\left(|u(t)|^{2}+\alpha^{2}\|u(t)\|^{2}\right) \leq \frac{1}{v \lambda_{1}}|g|^{2} .
$$


Thus applying Gronwall Lemma 2.1 to (3.9), (3.4) follows. Integrating (3.7) on $[t, t+$ 1], we find

$$
\begin{aligned}
& |u(t+1)|^{2}+\alpha^{2}\|u(t+1)\|^{2}+v \int_{t}^{t+1}\|u(s)\|^{2} d s \\
& \leq|u(t)|^{2}+\alpha^{2}\|u(t)\|^{2}+\frac{1}{v \lambda_{1}}|g|^{2},
\end{aligned}
$$

which, together with (3.4), gives (3.5). The proof is thus complete.

Lemma 3.3 If $g \in H$ and $u(t)$ is a weak solution of problem (3.1), then the following inequality holds:

$$
\int_{t}^{t+1}\left\|\partial_{t} u(s)\right\|_{D(A)^{\prime}}^{2} d s \leq C_{1}\left(|u(0)|^{2}+\alpha^{2}\|u(0)\|^{2}\right) e^{-\frac{v \lambda_{1}}{1+\lambda_{1} \alpha^{2}} t}+R_{1}^{2},
$$

where $C_{1}, R_{1}$ only depend on $v, \lambda_{1},|g|$, but are independent of $\alpha$.

Proof First, using the Poincaré inequality (2.3), we have

$$
\|g\|_{D(A)^{\prime}} \leq \frac{1}{\lambda_{1}}|g|
$$

and

$$
\|A u\|_{D(A)^{\prime}}=|u| \leq \frac{1}{\sqrt{\lambda_{1}}}\|u\| .
$$

Then using (3.5), we get

$$
\begin{aligned}
& v \int_{t}^{t+1}\|A u(s)\|_{D(A)^{\prime}}^{2} d s \leq \frac{v}{\lambda_{1}} \int_{t}^{t+1}\|u(s)\|^{2} d s \\
& \quad \leq \frac{1}{\lambda_{1}}\left(\left(|u(0)|^{2}+\alpha^{2}\|u(0)\|^{2}\right) e^{-\frac{v \lambda}{1+\lambda_{1} \alpha^{2}} t}+\frac{\left(1+\lambda_{1} \alpha^{2}\right)|g|^{2}}{v^{2} \lambda_{1}^{2}}+\frac{|g|^{2}}{v \lambda_{1}}\right)
\end{aligned}
$$

Second, we derive from inequality (2.2) that

$$
\|B(u, u)\|_{D(A)^{\prime}} \leq c|u|\|u\|,
$$

which, along with (3.4)-(3.5), yields

$$
\begin{aligned}
& \int_{t}^{t+1}\|B(u, u)\|_{D(A)^{\prime}}^{2} d s \leq c^{2} \sup _{s \in[t, t+1]}|u(s)|^{2} \int_{t}^{t+1}\|u(s)\|^{2} d s \\
& \leq \frac{c^{2}}{v}\left(|u(0)|^{2}+\alpha^{2}\|u(0)\|^{2}+\frac{\left(1+\lambda_{1} \alpha^{2}\right)|g|^{2}}{v^{2} \lambda_{1}^{2}}\right)
\end{aligned}
$$




$$
\times\left(\left(|u(0)|^{2}+\alpha^{2}\|u(0)\|^{2}\right) e^{-\frac{\nu \lambda}{1+\lambda_{1} \alpha^{2}} t}+\frac{\left(1+\lambda_{1} \alpha^{2}\right)|g|^{2}}{v^{2} \lambda_{1}^{2}}+\frac{|g|^{2}}{\nu \lambda_{1}}\right) .
$$

Obviously,

$$
\begin{aligned}
& \int_{t}^{t+1}\left\|\left(1+\alpha^{2} A\right) \partial_{t} u(s)\right\|_{D(A)^{\prime}}^{2} d s \leq v \int_{t}^{t+1}\|A u(s)\|_{D(A)^{\prime}}^{2} d s \\
& \quad+\int_{t}^{t+1}\|B(u, u)\|_{D(A)^{\prime}}^{2} d s+\|g\|_{D(A)^{\prime}}^{2} .
\end{aligned}
$$

Combining (3.12), (3.13), (3.15), then (3.16) becomes

$$
\begin{aligned}
& \int_{t}^{t+1}\left\|\left(1+\alpha^{2} A\right) \partial_{t} u(s)\right\|_{D(A)^{\prime}}^{2} d s \leq C_{\alpha}\left(|u(0)|^{2}+\alpha^{2}\|u(0)\|^{2}\right) e^{-\frac{\nu \lambda}{1+\lambda_{1} \alpha^{2}} t} \\
& \quad+R_{\alpha}^{2}
\end{aligned}
$$

where

$$
\left\{\begin{array}{l}
C_{\alpha}=\frac{1}{\lambda_{1}}+\frac{c^{2}}{v}\left(|u(0)|^{2}+\alpha^{2}\|u(0)\|^{2}+\frac{\left(1+\lambda_{1} \alpha^{2}\right)|g|^{2}}{v^{2} \lambda_{1}^{2}}\right) \\
R_{\alpha}^{2}=C_{\alpha}\left(\frac{\left(1+\lambda_{1} \alpha^{2}\right)|g|^{2}}{v^{2} \lambda_{1}^{2}}+\frac{|g|^{2}}{v \lambda_{1}}\right)+\frac{1}{\lambda_{1}^{2}} .
\end{array}\right.
$$

Since $\alpha \in(0,1)$, it follows

$$
\left\{\begin{array}{l}
C_{\alpha} \leq C_{1}=\frac{1}{\lambda_{1}}+\frac{c^{2}}{v}\left(|u(0)|^{2}+\|u(0)\|^{2}+\frac{\left(1+\lambda_{1}\right)|g|^{2}}{v^{2}}\right) \\
R_{\alpha}^{2} \leq R_{1}^{2}=C_{1}\left(\frac{\left(1+\lambda_{1}\right)|g|^{2}}{v^{2} \lambda_{1}^{2}}+\frac{|g|^{2}}{v \lambda_{1}}\right)+\frac{1}{\lambda_{1}^{2}}
\end{array}\right.
$$

Now by applying Lemma 2.3 and (3.17), the proof is thus complete.

Lemma 3.4 Let $g \in H$, then

(i) $\mathcal{K}_{\alpha}^{+} \subset \mathcal{F}_{+}^{b}$;

(ii) for any function $u(\cdot) \in \mathcal{K}_{\alpha}^{+}$, the translation group $\{T(h)\}$ is continuous in $\mathcal{F}_{+}^{b}$, and there holds that

$$
\|T(h) u(\cdot)\|_{\mathcal{F}_{+}^{b}}^{2} \leq C_{0}\left(|u(0)|^{2}+\|u(0)\|^{2}\right) e^{-\frac{\nu \lambda_{1}}{1+\lambda_{1} \alpha^{2}} h}+R_{0}^{2}, \quad \forall h \geq 0
$$

where $C_{0}, R_{0}$ only depend on $\nu, \lambda_{1},|g|$, but are independent of $\alpha$.

Proof The proof of (3.18) is clearly established by Lemmas 3.2-3.3. Let $u_{1}(s) \rightarrow$ $u_{2}(s)$ in $\mathcal{F}_{+}^{b}$. Then $u_{1}(s+h) \rightarrow u_{2}(s+h)$ in $\mathcal{F}_{+}^{b}$ and the translation group $\{T(h)\}$ is continuous in $\mathcal{F}_{+}^{b}$. 
Proposition 3.3 The trajectory space $\mathcal{K}_{\alpha}^{+} \subseteq \mathcal{F}_{+}^{b}$ is compact in the topology $\Theta_{+}^{\text {loc }}$.

Proof We consider an arbitrary sequence $\left\{u_{n}\right\} \subset \mathcal{K}_{\alpha}^{+}$, such that $u_{n} \rightarrow u$ in the topology $\Theta_{+}^{l o c}$ as $n \rightarrow \infty$, we need to prove $u \in \mathcal{K}_{\alpha}^{+}$. Due to $u_{n} \rightarrow u$ in the topology $\Theta_{+}^{l o c}$, by the definition of $\Theta_{+}^{l o c}$,

$u_{n} \rightarrow u$, weakly in $L^{2}(0, M ; V)$, and weakly-star in $L^{\infty}(0, M ; H), n \rightarrow \infty$, $\partial_{t} u_{n} \rightarrow \partial_{t} u$, weakly in $L^{2}\left(0, M ; D(A)^{\prime}\right), n \rightarrow \infty$.

Noting $\left\{u_{n}\right\} \subset \mathcal{K}_{\alpha}^{+}$and using Lemma $3.2, u_{n}$ is bounded in $L^{2}(0, M ; V) \cap$ $L^{\infty}(0, M ; H)$, and $u_{n} \rightarrow u$ strongly in $L^{2}(0, M ; H)$, then $\left\{u_{n}\right\}$ contains a subsequence $\left\{u_{n_{k}}\right\}$ such that

$$
\begin{aligned}
& B\left(u_{n_{k}}, u_{n_{k}}\right) \rightarrow B(u, u) \text { weakly in } L^{\frac{4}{3}}\left(0, M ; V^{\prime}\right), \\
& u_{n_{k}} \rightarrow u \text { weakly in } L^{2}(0, M ; V), \\
& u_{n_{k}} \rightarrow u \text { weakly-star in } L^{\infty}(0, M ; H) .
\end{aligned}
$$

Since $u_{n_{k}}(t) \in \mathcal{K}_{\alpha}^{+}$, we have

$$
\left(1+\alpha^{2} A\right) \partial_{t} u_{n_{k}}+v A u_{n_{k}}+B\left(u_{n_{k}}, u_{n_{k}}\right)=g(x)
$$

Taking limit of (3.22) and using (3.19)-(3.21), we have

$$
\left(1+\alpha^{2} A\right) \partial_{t} u+v A u+B(u, u)=g(x),
$$

then $u$ is a weak solution of problem (3.1), i.e., $u \in \mathcal{K}_{\alpha}^{+}$.

Proposition 3.4 The ball $B_{2 R_{0}}=\left\{u(\cdot) \mid u(\cdot) \in \mathcal{K}_{\alpha}^{+},\|T(h) u\|_{\mathcal{F}_{+}^{b}} \leq 2 R_{0}\right\}$ in $\mathcal{F}_{+}^{b}$ is an absorbing set of the semigroup $\{T(h)\} . B_{2 R_{0}}$ is compact in the topology $\Theta_{+}^{\text {loc }}$.

Proof According to Lemma 3.4, for any bounded set $B \subset \mathcal{K}_{\alpha}^{+}$, there exists a time $h_{1}>0$, such that as $h \geq h_{1}$,

$$
T(h) B \subset B_{2 R_{0}},
$$

which indicates that $B_{2 R_{0}}$ is an absorbing set of semigroup $T(h)$ on $\mathcal{K}_{\alpha}^{+}$. Next, since $B_{2 R_{0}} \subset \mathcal{K}_{\alpha}^{+} \subseteq \mathcal{F}_{+}^{b}$, and $B_{2 R_{0}}$ is closed, by Proposition $3.3, B_{2 R_{0}}$ is compact in the topology $\Theta_{+}^{l o c}$.

Definition 3.5 [6] A set $\mathcal{O}_{\alpha} \subset \mathcal{K}_{\alpha}^{+}$is said to be the trajectory attractor of equation, if

(i) $\mathcal{O}_{\alpha}$ is compact in the topology $\Theta_{+}^{l o c}$,

(ii) $\mathcal{O}_{\alpha}$ is strictly invariant, i.e., $T(h) \mathcal{O}_{\alpha}=\mathcal{O}_{\alpha}$ for all $h \geq 0$,

(iii) $\mathcal{O}_{\alpha}$ is a uniformly attracting set for $\mathcal{F}_{+}^{b}$. 
Theorem 3.5 If $g \in H$, then the translation semigroup $\{T(h)\}$ acting on $\mathcal{K}_{\alpha}^{+}$has a trajectory attractor $\mathcal{O}_{\alpha}$. The set $\mathcal{O}_{\alpha}$ is bounded in $\mathcal{F}_{+}^{b}$ and compact in the topology $\Theta_{+}^{l o c}$.

Proof Let

$$
\mathcal{O}_{\alpha}=B_{2 R_{0}}
$$

(i) By Proposition 3.4, we know that $\mathcal{O}_{\alpha}$ is compact in the topology of $\Theta_{+}^{l o c}$, and $T(h) \mathcal{O}_{\alpha} \subseteq \mathcal{O}_{\alpha}$ for all $h \geq h_{1}$.

(ii) On the other hand, let $u(t) \in B_{2 R_{0}}=\mathcal{O}_{\alpha}$, by the definition of time translation semigroup $\{T(h)\}$,

$$
u(t)=T(h) u(t-h),
$$

let $\tilde{u}(t)=u(t-h)$, obviously, $\tilde{u}$ is a weak solution of problem (3.1), i.e., $\tilde{u} \in \mathcal{O}_{\alpha}$, then there exists a time $t_{2}>0$, such that as $t \geq t_{2}$,

$$
\|\tilde{u}(t)\|_{\mathcal{F}_{+}^{b}} \leq 2 R_{0}
$$

hence, $\tilde{u} \in B_{2 R_{0}}=\mathcal{O}_{\alpha}$. Then from (3.24)-(3.25), we derive $u(t) \in T(h) B_{2 R_{0}}$ as $t \geq t_{2}$, i.e., $\mathcal{O}_{\alpha} \subseteq T(h) \mathcal{O}_{\alpha}$ as $t \geq t_{2}$. Letting $t_{0}=\max \left\{h_{1}, t_{2}\right\}$, as $t>t_{0}$, then we have $\mathcal{O}_{\alpha}=T(h) \mathcal{O}_{\alpha}$.

(iii) By Proposition 3.4, we know that $\mathcal{O}_{\alpha}$ is an absorbing set of $T(h)$. Then we prove that $\mathcal{O}_{\alpha}$ is a trajectory attractor of problem (3.1).

\section{Upper Semicontinuity of the Trajectory Attractors of 3D NS Equation}

Theorem 4.1 Let a sequence $\left\{u_{n}(t)\right\} \subset \mathcal{K}_{\alpha}^{+}, \alpha_{n} \rightarrow 0^{+}(n \rightarrow \infty)$, and $u_{n}(t) \rightarrow u(t)$ in the topology $\Theta_{+}^{\text {loc }}$ as $n \rightarrow \infty$. Then $u(t)$ is a weak solution of the $3 D$ Navier-Stokes equation such that $u$ satisfies the inequality (2.6), i.e., $u \in \mathcal{K}_{0}^{+}$, where $\mathcal{K}_{0}^{+}$is the trajectory space of problem (2.4).

Proof Since $u_{n}(t) \in \mathcal{K}_{\alpha}^{+}$, i.e., $u_{n}(t)$ is a weak solution of equation

$$
\left(1+\alpha_{n}^{2} A\right) \partial_{t} u_{n}+v A u_{n}+B\left(u_{n}, u_{n}\right)=g(x),
$$

and noticing (3.18), $u_{n}(t)$ satisfies

$$
\begin{aligned}
& \text { ess } \sup _{t \geq 0}\left|u_{n}(t)\right|^{2} \leq C, \\
& \sup _{t \geq 0} \int_{t}^{t+1}\left\|u_{n}(\tau)\right\|^{2} d \tau \leq C,
\end{aligned}
$$




$$
\sup _{t \geq 0} \int_{t}^{t+1}\left\|\partial_{t} u_{n}(\tau)\right\|^{2} d \tau \leq C
$$

Since $\partial_{t} u_{n} \rightarrow \partial_{t} u \quad(n \rightarrow \infty)$ weakly in $L^{2}\left(0, M ; D(A)^{\prime}\right)$ and using Lemma 2.4, we have

$$
\left(1+\alpha_{n}^{2} A\right) \partial_{t} u_{n} \rightarrow \partial_{t} u(n \rightarrow \infty) \text { weakly in } L^{2}\left(0, M ; D(A)^{\prime}\right)
$$

Since $u_{n} \rightarrow u(n \rightarrow \infty)$ weakly in $L^{2}(0, M ; V)$, then $A u_{n} \rightarrow A u(n \rightarrow \infty)$ weakly in $L^{2}\left(0, M ; V^{\prime}\right)$. Obviously,

$$
A u_{n} \rightarrow A u(n \rightarrow \infty) \text { weakly in } L^{2}\left(0, M ; D(A)^{\prime}\right) .
$$

By (2.2), (4.2) and (4.3), we have

$$
\int_{0}^{M}\left\|B\left(u_{n}, u_{n}\right)\right\|_{D(A)^{\prime}}^{2} d s \leq c \int_{0}^{M}\left(\left|u_{n}\right|\left\|u_{n}\right\|\right)^{2} d s \leq C
$$

then

$$
B\left(u_{n}, u_{n}\right) \rightarrow B(u, u)(n \rightarrow \infty) \text { weakly in } L^{2}\left(0, M ; D(A)^{\prime}\right) .
$$

Combining with (4.5)-(4.7), Eq. (4.1) converges to

$$
\partial_{t} u+v A u+B(u, u)=g(x), \quad(n \rightarrow \infty) \text { weakly in } L^{2}\left(0, M ; D(A)^{\prime}\right) .
$$

Next, we prove $u$ satisfies the enery inequality (2.6). Since $u_{n}(t) \rightarrow u(t)$ in the topology $\Theta_{+}^{l o c}$ as $n \rightarrow \infty$, by the Aubin Theorem, there exists a subsequence $u_{n}$ which we still denote by $u_{n}$ such that

$$
\left\|u_{n}(t)-u(t)\right\|_{L^{2}(0, M ; H)}^{2}=\int_{0}^{M}\left|u_{n}(t)-u(t)\right|^{2} d t \rightarrow 0, \quad n \rightarrow \infty .
$$

Note that $u_{n}(t)$ satisfies the energy equality

$$
\begin{aligned}
& -\frac{1}{2} \int_{0}^{M}\left(\left|u_{n}(t)\right|^{2}+\alpha_{n}^{2}\left\|u_{n}(t)\right\|^{2}\right) \psi^{\prime}(t) d t+v \int_{0}^{M}\left\|u_{n}(t)\right\|^{2} \psi(t) d t \\
& =\int_{0}^{M}\left(g, u_{n}(t)\right) \psi^{\prime}(t) d t, \quad \forall \psi^{\prime}(t) \in C_{0}^{\infty}(0, M) .
\end{aligned}
$$


Applying (4.9) and the Hölder inequality, we have

$$
\begin{aligned}
& \int_{0}^{M}\left|u_{n}(t)\right|^{2} \psi^{\prime}(t) d t-\int_{0}^{M}|u(t)|^{2} \psi^{\prime}(t) d t \\
& \leq \sup _{t \in[0, M]}\left|\psi^{\prime}(t)\right| \int_{0}^{M}\left(\left|u_{n}(t)\right|+|u(t)|\right)\left(\left|u_{n}(t)\right|-|u(t)|\right) d t \\
& \leq \sup _{t \in[0, M]}\left|\psi^{\prime}(t)\right|\left(2 \int_{0}^{M}\left(\left|u_{n}(t)\right|^{2}+|u(t)|^{2}\right) d t\right)^{\frac{1}{2}} \\
& \quad \times\left(\int_{0}^{M}\left|u_{n}(t)-u(t)\right|^{2} d t\right)^{\frac{1}{2}} \rightarrow 0, n \rightarrow \infty
\end{aligned}
$$

Similarly,

$$
\begin{aligned}
& \left|\int_{0}^{M}\left(g, u_{n}(t)\right) \psi^{\prime}(t) d t-\int_{0}^{M}(g, u(t)) \psi^{\prime}(t) d t\right| \leq \int_{0}^{M}|g|\left|u_{n}(t)-u(t)\right| \psi^{\prime}(t) d t \\
& \quad \leq\left(\int_{0}^{M}\left(|g| \psi^{\prime}(t)\right)^{2} d t\right)^{\frac{1}{2}}\left(\int_{0}^{M}\left|u_{n}(t)-u(t)\right|^{2} d t\right)^{\frac{1}{2}} \rightarrow 0, \quad n \rightarrow \infty
\end{aligned}
$$

Since $u_{n} \sqrt{\psi} \rightarrow u \sqrt{\psi}$ weakly in $L^{2}(0, M ; V)$, i.e., for any $w \in V^{\prime}$,

$$
\int_{0}^{M}\langle u \sqrt{\psi}, w\rangle d t=\lim _{n \rightarrow \infty} \int_{0}^{M}\left\langle u_{n} \sqrt{\psi}, w\right\rangle d t .
$$

Letting $w=u \sqrt{\psi}$, then

$$
\int_{0}^{M}\|u \sqrt{\psi}\|^{2} d t \leq \lim _{n \rightarrow \infty}\left(\int_{0}^{M}\left\|u_{n} \sqrt{\psi}\right\|^{2} d t\right)^{\frac{1}{2}}\left(\int_{0}^{M}\|u \sqrt{\psi}\|^{2} d t\right)^{\frac{1}{2}}
$$

and $\psi(t)$ is independent of $x$, we obtain

$$
v \int_{0}^{M}\|u(t)\|^{2} \psi d t \leq v \lim _{n \rightarrow \infty} \int_{0}^{M}\left\|u_{n}(t)\right\|^{2} \psi d t .
$$

Similarly, letting $\psi^{\prime}$ replace $\psi$, and using (4.3), we have

$$
\lim _{n \rightarrow \infty} \int_{0}^{M}\left\|u_{n}(t)\right\|^{2} \psi^{\prime}(t) d t \leq C
$$

then

$$
\lim _{n \rightarrow \infty} \alpha_{n}^{2} \int_{0}^{M}\left\|u_{n}(t)\right\|^{2} \psi^{\prime}(t) d t=0
$$


Taking limit on both sides of equation (4.10), and using (4.11)-(4.14), we have

$$
-\frac{1}{2} \int_{0}^{M}|u(t)|^{2} \psi^{\prime}(t) d t+v \int_{0}^{M}\|u(t)\|^{2} \psi(t) d t \leq \int_{0}^{M}(g, u(t)) \psi^{\prime}(t) d t .
$$

Thus $u \in \mathcal{K}_{0}^{+}$, and the proof is now complete.

Theorem 4.2 Let $\mathbb{B}_{\alpha}=\left\{\left.u(t)|| u(t)\right|^{2}+\alpha^{2}\|u(t)\|^{2} \leq \frac{\left(1+\lambda_{1} \alpha^{2}\right)|g|^{2}}{v^{2} \lambda_{1}^{2}}\right\}, 0<\alpha \leq 1$, be the bounded sets of solutions of $3 D$ Navier-Stokes-Voight equation (3.1). Then the following convergence holds:

$$
T(h) \mathbb{B}_{\alpha} \rightarrow \mathcal{O}_{0} \text { in the topology } \Theta_{+}^{\text {loc }} \text { as } h \rightarrow+\infty, \alpha \rightarrow 0^{+},
$$

where $\mathcal{O}_{0}$ is the trajectory attractor of $3 D$ incompressible Navier-Stokes equation (2.4).

Proof There exists a sequence $\alpha_{n} \rightarrow 0^{+}$, as $n \rightarrow+\infty$. Let $u^{\alpha_{n}}(t) \in \mathbb{B}_{\alpha}, u^{\alpha_{n}}(t)$ is the solutions of 3D Navier-Stokes-Voight equation (3.1), i.e., $u^{\alpha_{n}}(t) \in \mathcal{K}_{\alpha}^{+}$,

$$
T(h) u^{\alpha_{n}}(t)=u^{\alpha_{n}}(t+h) \text {, since } T(h) \mathcal{K}_{\alpha}^{+} \subseteq \mathcal{K}_{\alpha}^{+} \text {, hence } u^{\alpha_{n}}(t+h) \in \mathcal{K}_{\alpha}^{+},
$$

i.e.,

$$
\left(1+\alpha_{n}^{2} A\right) \partial_{t} u^{\alpha_{n}}(t+h)+v A u^{\alpha_{n}}(t+h)+B\left(u^{\alpha_{n}}(t+h), u^{\alpha_{n}}(t+h)\right)=g(x) .
$$

Since $u^{\alpha_{n}}(t+h) \in \mathcal{K}_{\alpha}^{+}$, then

$$
\left\|u^{\alpha_{n}}(t+h)\right\|_{\mathcal{F}_{+}^{b}} \leq R
$$

By Theorem 4.1, there exists $u(t+h)$ in $\mathcal{F}_{+}^{l o c}$, such that $u^{\alpha_{n}}(t+h) \rightarrow u(t+h)$ in the topology $\Theta_{+}^{l o c}$ as $n \rightarrow+\infty$, and $u(t+h)$ is a weak solution of 3D Navier-Stokes equation, then $u(t+h)$ solves

$$
\partial_{t} u(t+h)+v A u(t+h)+B(u(t+h), u(t+h))=g(x) .
$$

There exists a sequence $h_{n} \rightarrow+\infty$, as $n \rightarrow+\infty$. Next we prove $\lim _{n \rightarrow+\infty} u\left(t+h_{n}\right) \in$ $\mathcal{O}_{0}$. (4.17) means that

$$
\begin{aligned}
\left\|u\left(t+h_{n}\right)\right\|_{\mathcal{F}_{+}^{b}}= & \sup _{t \geq-h_{n}}|u(t)|+\left(\sup _{t \geq-h_{n}} \int_{t}^{t+1}\|u(s)\|^{2} d s\right)^{\frac{1}{2}} \\
& +\left(\sup _{t \geq-h_{n}} \int_{t}^{t+1}\left\|\partial_{s} u(s)\right\|_{D(A)^{\prime}}^{2} d s\right)^{\frac{1}{2}} \leq R
\end{aligned}
$$


hence

$$
\begin{aligned}
& \lim _{n \rightarrow+\infty}\left\|u\left(t+h_{n}\right)\right\|_{\mathcal{F}_{+}^{b}}=\sup _{t \in \mathbb{R}}|u(t)|+\left(\sup _{t \in \mathbb{R}} \int_{t}^{t+1}\|u(s)\|^{2} d s\right)^{\frac{1}{2}} \\
& \quad+\left(\sup _{t \in \mathbb{R}} \int_{t}^{t+1}\left\|\partial_{s} u(s)\right\|_{D(A)^{\prime}}^{2} d s\right)^{\frac{1}{2}} \leq R,
\end{aligned}
$$

i.e., for all $t \in \mathbb{R}$,

$$
\begin{gathered}
\lim _{n \rightarrow+\infty} u\left(t+h_{n}\right)=u(t) \in \mathcal{F}^{b}=L^{2}(\mathbb{R}, V) \\
\cap L^{\infty}(\mathbb{R}, H) \cap\left\{u \mid \partial_{t} u \in L^{2}\left(\mathbb{R}, D(A)^{\prime}\right)\right\},
\end{gathered}
$$

and $u(t)$ is a weak solution of 3D incompressible Navier-Stokes equation, i.e., $u(t) \in$ $\mathcal{O}_{0}$.

From the above argument, we have proved the following proposition.

Proposition 4.3 According to the definition of $\mathcal{O}_{\alpha}$, the following convergence holds:

$$
\mathcal{O}_{\alpha} \rightarrow \mathcal{O}_{0} \text { in the topology } \Theta_{+}^{\text {loc }} \text { as } \alpha \rightarrow 0^{+} \text {. }
$$

The following theorem concerns the upper semicontinuity of trajectory attractors of 3D Navier-Stokes equation when the regular term $\alpha^{2} \Delta u_{t}$ in NSV equation is considered as its a perturbative term. Denote by $\operatorname{dist}_{X}\left(B_{1}, B_{2}\right)$ the Hausdorff semidistance in space $X$ between $B_{1}$ and $B_{2}$, i.e.,

$$
\operatorname{dist}_{X}\left(B_{1}, B_{2}\right)=\sup _{x \in B_{1}} \inf _{y \in B_{2}} d_{X}(x, y), \quad B_{1}, B_{2} \subset X .
$$

Theorem 4.4 Assume that $\mathcal{O}_{\alpha}, \mathcal{O}_{0}$ are the trajectory attractors of $3 D$ NavierStokes-Voight equation and Navier-Stokes equation respectively. Then the following convergence holds:

$$
\lim _{\alpha \rightarrow 0^{+}} \operatorname{dist}_{L^{2}\left(0, M ; H^{-\delta}\right)}\left(\mathcal{O}_{\alpha}, \mathcal{O}_{0}\right)=0
$$

here $\delta \in(0,1]$.

Proof Let $u^{\alpha} \in \mathcal{O}_{\alpha}$, by Proposition 4.3, there exists $u(t) \in \mathcal{O}_{0}$ such that $u^{\alpha} \rightarrow u(t)$ weakly in $L^{2}(0, M ; V)$ as $\alpha \rightarrow 0^{+}$, i.e., $\forall \varphi \in V^{\prime}, u^{\alpha}, u \in V$, there holds that

$$
\begin{aligned}
& \lim _{\alpha \rightarrow 0^{+}} \int_{0}^{M}\left\langle u^{\alpha}(t)-u(t), \varphi\right\rangle d t=0 \\
& \int_{0}^{M}\left\langle u^{\alpha}(t)-u(t), \varphi\right\rangle d t=\int_{0}^{M}\left(A^{\frac{1}{2}}\left(u^{\alpha}(t)-u(t)\right), A^{\frac{1}{2}} \varphi\right) d t
\end{aligned}
$$




$$
=\int_{0}^{M}\left(u^{\alpha}(t)-u(t), A \varphi\right) d t
$$

Since $\varphi \in V^{\prime}$ without loss of generality, letting $\varphi=A^{-1}\left(u^{\alpha}(t)-u(t)\right)$, it follows from (4.24), (4.25) that

$$
\lim _{\alpha \rightarrow 0^{+}} \int_{0}^{M}\left|u^{\alpha}(t)-u(t)\right|_{H}^{2} d t=0,
$$

Let $u^{\alpha} \in \mathcal{O}_{\alpha}, u(t) \in \mathcal{O}_{0}, w(t)=u^{\alpha}-u$, then $w(t)$ satisfies

$$
\partial_{t} w+\alpha^{2} A u_{t}^{\alpha}+v A w+B\left(u^{\alpha}, w\right)+B(w, u)=0,
$$

then

$$
\left\|\partial_{t} w\right\|_{L^{2}\left(0, M ; D(A)^{\prime}\right)} \leq\left\|\alpha^{2} A u_{t}^{\alpha}+v A w+B\left(u^{\alpha}, w\right)+B(w, u)\right\|_{L^{2}\left(0, M ; D(A)^{\prime}\right)} .
$$

According to (2.2) and the interpolation inequality, we have

$$
\begin{aligned}
& \left\|B\left(u^{\alpha}, w\right)\right\|_{L^{2}\left(0, M ; D(A)^{\prime}\right)}^{2} \leq c \int_{0}^{M}\left|u^{\alpha}\right|\left\|u^{\alpha}\right\||w|^{2} d s \leq c\left(\int_{0}^{M}\left|u^{\alpha}\right|^{2}|w|^{2} d s\right)^{\frac{1}{2}} \\
& \quad \times\left(\int_{0}^{M}\left\|u^{\alpha}\right\|^{2}|w|^{2} d s\right)^{\frac{1}{2}} \\
& \quad \leq c\left(\sup _{s \in[0, M]}\left|u^{\alpha}\right|^{2} \int_{0}^{M}|w|^{2} d s\right)^{\frac{1}{2}} \times\left(\sup _{s \in[0, M]}|w|^{2} \int_{0}^{M}\left\|u^{\alpha}\right\|^{2} d s\right)^{\frac{1}{2}} \cdot
\end{aligned}
$$

Since $|w|^{2}=\left|u^{\alpha}-u\right|^{2} \leq 2\left(\left|u^{\alpha}\right|^{2}+|u|^{2}\right)$, and using (3.4)-(3.5), we get

$$
\begin{aligned}
& \max \left\{\sup _{s \in[0, M]}\left|u^{\alpha}\right|^{2}, \sup _{s \in[0, M]}|w|^{2}, \int_{0}^{M}\left\|u^{\alpha}\right\|^{2} d s\right\} \\
& \leq 2\left(\left(|u(0)|^{2}+\|u(0)\|^{2}\right)+\frac{\left(1+\lambda_{1}\right)|g|^{2}}{v^{2} \lambda_{1}^{2}}+\frac{|g|^{2}}{v \lambda_{1}}\right) .
\end{aligned}
$$

Then (4.29) becomes

$$
\left\|B\left(u^{\alpha}, w\right)\right\|_{L^{2}\left(0, M ; D(A)^{\prime}\right)}^{2} \leq C\left(\int_{0}^{M}|w|^{2} d s\right)^{\frac{1}{2}} .
$$


Similarly to $B(w, u)$, we obtain

$$
\|B(w, u)\|_{L^{2}\left(0, M ; D(A)^{\prime}\right)}^{2} \leq C\left(\int_{0}^{M}|w|^{2} d s\right)^{\frac{1}{2}},
$$

and

$$
v\|A w\|_{L^{2}\left(0, M ; D(A)^{\prime}\right)}^{2}=v \int_{0}^{M}|w|^{2} d s .
$$

By Lemma 2.4, taking $f(t)=u_{t}^{\alpha}$, we have

$$
\lim _{\alpha \rightarrow 0^{+}} \alpha^{2}\left\|A u_{t}^{\alpha}\right\|_{L^{2}(0, M ; H)}=0 .
$$

Obviously, $\left\|A u_{t}^{\alpha}\right\|_{D(A)^{\prime}}=\left|u_{t}^{\alpha}\right| \leq\left|A u_{t}^{\alpha}\right|$, then

$$
\lim _{\alpha \rightarrow 0^{+}} \alpha^{2}\left\|A u_{t}^{\alpha}\right\|_{L^{2}\left(0, M ; D(A)^{\prime}\right)}=0 .
$$

Combining (4.31)-(4.34) with (4.26), we obtain

$$
\lim _{\alpha \rightarrow 0^{+}}\left\|\partial_{t} w\right\|_{L^{2}\left(0, M ; D(A)^{\prime}\right)}=0 .
$$

Letting $E_{1}=H, E=H^{-\delta}, E_{0}=D(A)^{\prime}, p_{1}=2, p_{0}=2$, and using Lemma 2.2, we can prove (4.23).

Acknowledgements This paper was supported in part by the NNSF of China (No.11671075) and fundamental research funds for the central universities (No.2232019D3-43).

Open Access This article is distributed under the terms of the Creative Commons Attribution 4.0 International License (http://creativecommons.org/licenses/by/4.0/), which permits unrestricted use, distribution, and reproduction in any medium, provided you give appropriate credit to the original author(s) and the source, provide a link to the Creative Commons license, and indicate if changes were made.

\section{References}

1. Bronzi, A.C., Rosa, R.M.S.: On the convergence of statistical solutions of the 3D Navier-Stokes- $\alpha$ model as $\alpha$ vanishes. Discret. Contin. Dyn. Syst. 34(1), 19-49 (2014)

2. Chen, S., Holm, D.D., Margolin, L.G., Zhang, R.: Direct numerical simulations of the Navier-Stokes- $\alpha$ model. Physica D 133, 66-83 (1999)

3. Chepyzhov, V.V., Gatti, S., Grasselli, M., Miranville, A., Pata, V.: Trajectory and global attractors for evolution equations with memory. Appl. Math. Lett. 19, 87-96 (2006)

4. Chepyzhov, V.V., Titi, E.S., Vishik, M.I.: On Convergence of Trajectory Attractors of 3D NavierStokes- $\alpha$ Model as $\alpha$ Approaches 0. Russian Academy of Sciences, (DoM) and London Mathematical Society (2007)

5. Chepyzhov, V.V., Titi, E.S., Vishik, M.I.: On the convergence of solutions of the Leray- $\alpha$ model to the trajectory attractor of the 3D Navier-Stokes system. Discret. Contin. Dyn. Syst. 17, 481-500 (2007) 
6. Chepyzhov, V.V., Vishik, M.I.: Attractors for Equations of Mathematical Physics, AMS, vol. 49. Collouium Publications, American Mathematical Society, Providence, RI (2002)

7. Chepyzhov, V.V., Vishik, M.I.: Trajectory attractors for dissipative 2D Euler and Navier-Stokes equations. Russ. J. Math. Phys. 15(2), 156-170 (2008)

8. Deugoue, G.: Approximation of the trajectory attractor of the 3D MHD system. Commun. Pure Appl. Anal. 12(5), 2119-2144 (2013)

9. Foias, C., Holm, D.D., Titi, E.S.: The Navier-Stokes-alpha model of fluid turbulence. Physica D 153, 505-519 (2001)

10. Foias, C., Holm, D.D., Titi, E.S.: The three dimensional viscous Camassa-Holm equations, and their relation to the Navier-Stokes equations and turbulence theory. J. Dyn. Differ. Equ. 14, 1-35 (2002)

11. Frigeri, S., Grasselli, M.: Global and trajectory attractors for a nonlocal Cahn-Hilliard-Navier-Stokes system. J. Dyn. Differ. Equ. 24, 827-856 (2012)

12. Frigeri, S., Rocca, E.: Trajectory attractors for the Sun-Liu model for nematic liquid crystals in 3D. Nonlinearity 26(4), 933-958 (2013)

13. Gal, C.G., Grassellt, M.: Trajectory attractors for binary fluid mixtures in 3D. Chin. Ann. Math. 31B(5), 655-678 (2010)

14. Gorban, N.V., Kapustyan, O.V., Kasyanov, P.O.: Uniform trajectory attractor for non-autonomous reaction-diffusion equations with Carath odory's nonlinearity. Nonlinear Anal. 98, 13-26 (2014)

15. Grasselli, M., Schimperna, G., Zelik, S.: Trajectory and smooth attractors for Cahn-Hilliard equations with inertial term. Nonlinearity 23(3), 707-738 (2010)

16. Guo, B.L., Huang, D.W.: Existence of weak solutions and trajectory attractors for the moist atmospheric equations in geophysics. J. Math. Phys. 47(8), 083508, 1-23 (2006)

17. Hetzer, G.: Trajectory attractors of energy balance climate models with Bio-Feedback. Differ. Equ. Appl. 3(4), 565-579 (2011)

18. Kalantarov, V.K., Titi, E.S.: Global attractors and determining modes for the 3D Navier-Stokes-Voight equations. Chin. Ann. Math. 30B(6), 697-714 (2009)

19. Mielke, A., Zelik, S.V.: Infinite-dimensional trajectory attractors of elliptic boundary-value problems in cylindrical domains. Russ. Math. Surv. 57(4), 753-784 (2002)

20. Qin, Y.: Analytic Inequalities and their Applications in PDEs. Springer, Berlin (2017)

21. Zelati, M.C., Gal, C.G.: Singular limits of Voight models in fluid dynamics. J. Math. Fluid Mech. 17, 233-259 (2005)

22. Zhao, C.D., Zhou, S.F.: Pullback trajectory attractors for evolution equations and application to 3D incompressible non-Newtonian fluid. Nonlinearity 21, 1691-1717 (2008)

Publisher's Note Springer Nature remains neutral with regard to jurisdictional claims in published maps and institutional affiliations. 